\title{
What is the Right Theory for Anderson Localization of Light? An Experimental Test
}

\author{
Walter Schirmacher, ${ }^{1,2,3}$ Behnam Abaie, ${ }^{4}$ Arash Mafi, ${ }^{4}$ Giancarlo Ruocco, ${ }^{1,2}$ and Marco Leonetti ${ }^{1,5}$ \\ ${ }^{1}$ Center for Life Nano Science @ Sapienza, Isituto Italiano di Tecnologia, Viale Regina Elena, 291, I-00161 Roma, Italia \\ ${ }^{2}$ Dipartimento di Fisica, Universitá “La Sapienza," Piazzale Aldo Moro, 5, I-00185 Roma, Italia \\ ${ }^{3}$ Institut für Physik, Universität Mainz, Staudinger Weg 7, D-55099 Mainz, Germany \\ ${ }^{4}$ Department of Physics and Astronomy and Center for High Technology Materials, \\ University of New Mexico, Albuquerque, New Mexico 87131, USA \\ ${ }^{5}$ CNR NANOTEC, Istituto di Nanotechologia, I-73100 Lecce, Italia
}

(Received 29 March 2017; published 5 February 2018)

\begin{abstract}
Anderson localization of light is traditionally described in analogy to electrons in a random potential. Within this description, the random potential depends on the wavelength of the incident light. For transverse Anderson localization, this leads to the prediction that the distribution of localization lengthsand, hence, its average — strongly depends on the wavelength. In an alternative description, in terms of a spatially fluctuating electric modulus, this is not the case. Here, we report on an experimentum crucis in order to investigate the validity of the two conflicting theories using optical samples exhibiting transverse Anderson localization. We do not find any dependence of the observed average localization radii on the light wavelength. We conclude that the modulus-type description is the correct one and not the potentialtype one. We corroborate this by showing that in the derivation of the traditional potential-type theory, a term in the wave equation has been tacitly neglected. In our new modulus-type theory, the wave equation is exact. We check the consistency of the new theory with our data using the nonlinear sigma model. We comment on the consequences for the general case of three-dimensional disorder.
\end{abstract}

DOI: $10.1103 /$ PhysRevLett.120.067401

Anderson localization, i.e., the possibility of an arrest of the motion of an electronic wave packet in a disordered environment, has fascinated researchers since the appearance of Anderson's 1958 article [1]. Later, it became clear that this phenomenon is due to destructive interference of recurrent scattering paths and led-via the one-parameter scaling hypothesis $[2,3]$ and the mapping to the nonlinear sigma model [4-7] — to the conclusion that in disordered one- and two-dimensional systems, the waves are always localized.

Anderson localization of light [8] has gained much attention recently in wave optics due to a large number of possible applications reaching from solar cells to endoscopic fibers [9-11].

In the description of possible localization of light by means of the nonlinear-sigma-model theory, John [12-14] adopted the same structure of a classical wave equation with disorder as in his sound-wave theory $[15,16]$, namely, a fluctuating coefficient of the double time derivative. In the time-Fourier-transformed version of the wave equation, this version had the attractive feature that a one-to-one mapping to the Schrödinger equation of an electron in a random potential could be established, so most of the results of the theory for the electronic Anderson localization could be taken over [12-14,17-19]. We call this approach the "potential-type" description (PT).

On the other hand, in an alternative formulation used successfully for the vibrational anomalies in glasses
[20-22], the disorder enters the coefficient of the spatial derivatives, which, in the case of sound waves, is the elastic modulus, and in the case of electromagnetic waves, the dielectric modulus $1 / \epsilon(\mathbf{r})$. We call this the "modulus-type" description (MT).

While until now there is no clear experimental evidence for Anderson localization of light in three-dimensional disordered optical materials [23,24], in optical systems with restricted dimensionality [25,26], one has nowadays evidence for Anderson localization, in particular, in optical fibers with transverse disorder [11,27-29].

In fibers composed of microfibers with different dielectric constants, the presence of transverse localization leads to the existence of channels with the diameter of the transverse localization length, which transmit light like in a microwaveguide. As the localized modes have been proven to be of single-mode character [30], such fibers are extremely useful for transfer of multiple information, e.g., for endoscopy.

The theoretical description of transverse Anderson localization in fibers with transverse disorder [26,28,31] followed the potential-type approach. This description predicts a rather strong dependence of the average localization lengths on the wavelength of the applied light [31].

In the present contribution, we show that this description is not consistent with our experimental observation. We have measured the average localization lengths of fibers with transverse disorder as a function of the light 
wavelength and do not find any change with wavelength. Motivated by this observation, we adopted the modulustype approach to disorder and found perfect consistency with the experiments. We conclude that the modulus-type description is the correct theory for Anderson localization of light. A further argument against the potential-type approach is that it predicts the fibers to be opaque in the longitudinal $(z)$ direction. The modulus-type model predicts the scattering mean free path to diverge as the inverse third power of the transverse wave number (proportional to the angle between the ray and the fiber axis) so that transparency in the direction of the fiber axis is recovered.

In the frequency regime (with frequency variable $\omega=2 \pi c_{0} / \lambda \sqrt{\langle\epsilon\rangle}=c_{0} k_{0} / \sqrt{\langle\epsilon\rangle}$, where $c_{0}$ is the vacuum light speed, $\lambda$ is the wavelength, $k_{0}=2 \pi / \lambda$ is the wave number, and $\langle\epsilon\rangle$ the average permittivity), the two conflicting stochastic wave equations, which are both considered to be derived from Maxwell's equations with inhomogeneous permittivity, take the form

$$
\begin{array}{rl}
{\left[\tilde{\epsilon}(\mathbf{r}) k_{0}^{2}+\nabla^{2}\right] \mathbf{E}(\mathbf{r}, \omega)=0} & \mathrm{PT}, \\
\left(k_{0}^{2}-\nabla \times \frac{1}{\tilde{\epsilon}(\mathbf{r})} \nabla \times\right) \mathbf{B}(\mathbf{r}, \omega) & =0 \quad \mathrm{MT},
\end{array}
$$

where $\mathbf{E}$ and $\mathbf{B}$ are the electric and magnetic field vectors, and $\tilde{\epsilon}(\mathbf{r})=\epsilon(\mathbf{r}) /\langle\epsilon\rangle$ denotes the relative fluctuations of the dielectric constant.

In the case of transverse disorder, $\epsilon$ fluctuates only in the $x, y$ direction, so one can perform a Fourier transform with respect to the $z$ direction $\left(\partial / \partial z \rightarrow i k_{z}\right)$ to obtain

$$
\begin{array}{rl}
{\left[\mathrm{E}+k_{0}^{2} \Delta \tilde{\epsilon}(\boldsymbol{\rho})+\nabla_{\boldsymbol{\rho}}^{2}\right] \mathbf{E}\left(k_{z}, \boldsymbol{\rho}, \omega\right)=0} & \mathrm{PT}, \\
\left(\mathrm{E}-\nabla_{\boldsymbol{\rho}} \times \frac{1}{\tilde{\epsilon}(\boldsymbol{\rho})} \nabla_{\boldsymbol{\rho}} \times\right) \mathbf{B}\left(k_{z}, \boldsymbol{\rho}, \omega\right)=0 \quad \mathrm{MT},
\end{array}
$$

with $\boldsymbol{\rho}=x \mathbf{e}_{x}+y \mathbf{e}_{y}$ and $\Delta \tilde{\epsilon}(\boldsymbol{\rho})=\tilde{\epsilon}(\boldsymbol{\rho})-1$. Here, we have introduced the spectral parameter (eigenvalue) $\mathrm{E}=k_{\perp}^{2}=$ $k_{0}^{2}-k_{z}^{2}=k_{0}^{2} \sin (\theta)^{2}$, where $\theta$ is the azimuthal angle (we use sans-serif $E$ for the spectral parameter and boldface $\mathbf{E}$ for the electrical field).

We note that the two wave equations are not consistent with respect to each other because in the PT equation, the wave number $k_{0}=2 \pi / \lambda$ appears as an external parameter in front of the fluctuating permittivity, whereas in the MT version, $k_{0}$ enters only via the spectral parameter $\mathrm{E}$.

We further note that one can establish a mathematical analogy between the MT version of Eq. (2) and a twodimensional wave system with modulus-type disorder via the correspondence $\mathrm{E} \leftrightarrow-\omega^{2}$. In such a system, the disorder leads to a Rayleigh law for the mean free path $\ell(\omega) \propto \omega^{-3}$ for $\omega \rightarrow 0$, as shown rigorously by Ganter and Schirmacher [32]. Transforming this back to the transversedisordered system, we have $\ell(\mathrm{E}) \propto \mathrm{E}^{-3 / 2} \propto \theta^{-3}$ for $\mathrm{E} \rightarrow 0$. This indicates that for $\theta \rightarrow 0$, the electromagnetic waves are not affected by the disorder. In other words, the rays directed strictly in the $z$ direction are not scattered. This agrees with the physically reasonable fact that the samples are transparent in the $z$ direction, in which there is no disorder. On the other hand, according to the PT version of Eq. (2), which is equivalent to the Schrödinger equation of an electron in a random potential, there is no diminishing of the scattering for $E \rightarrow 0$. This agrees to our fieldtheoretical calculations in Fig. 3. This means that the PT wave equation predicts that the transversely disordered fibers would be opaque in the $z$ direction, as said in the introduction.

In the paraxial limit, $\theta \rightarrow 0 \mathrm{E}$ can approximated by $-2 k_{0} \Delta k_{z}$, where $\Delta k_{z}=k_{z}-k_{0}$ is the Fourier wave number corresponding to the $z$ dependence of the envelopes of the electromagnetic fields (paraxial approximation). Transformed back to the $z$ coordinate, one then obtains the paraxial Helmholtz equation, the PT version of which has been used in Refs. [26-28,31] for a numerical calculation of the localization properties of transverse-disordered optical fibers. In Fig. 1(a), we have reproduced the $z$ dependence of the average localization lengths $\xi(z)$ obtained by such a simulation [31] for two different light wavelengths $\lambda$, which saturate for large $z$ at the localization length $\xi(z=\infty) \equiv \xi_{\infty}$. The strong dependence on the wavelength is striking.

In order to check whether this behavior predicted by the PT theory is realistic, we have taken samples composed of microfibers with different permittivity (polystyrene, PS $\epsilon_{\mathrm{PS}} / \epsilon_{0}=1.59$, polymethylmethacrylate, PMMA, $\left.\epsilon_{\mathrm{PMMA}} / \epsilon_{0}=1.49\right)$ fabricated as described in Refs. [28,33,34] in which transverse localization is observed [28,31,35,36]. We measured the localization length in such devices by injecting a focused (order of a micrometer) monochromatic light at the fiber input tip while monitoring the total fiber output. The average extent of the output intensity pattern is determined by the

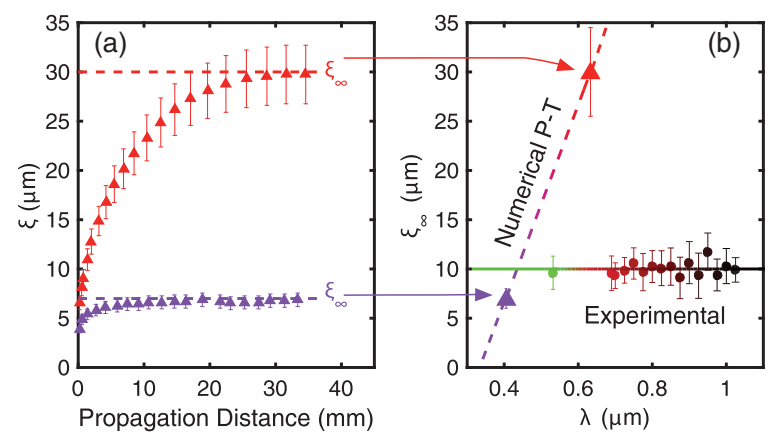

FIG. 1. (a) Average localization length $\xi(z)$ as a function of the distance $z$ along the fiber for the two light wavelengths $\lambda=$ $0.4 \mu \mathrm{m}$ and $\lambda=0.63 \mu \mathrm{m}$ from the simulation of Karbasi et al. [31] based on the potential-type theory (dashed line is to guide the eyes). (b) Measured averaged localization length of fibers with transverse disorder as a function of the incident-laser wavelength (full circles) compared with the two values $\xi_{\infty} \equiv \xi(z=\infty)$ of panel (a) (full triangles). Full line is to guide the eyes. 


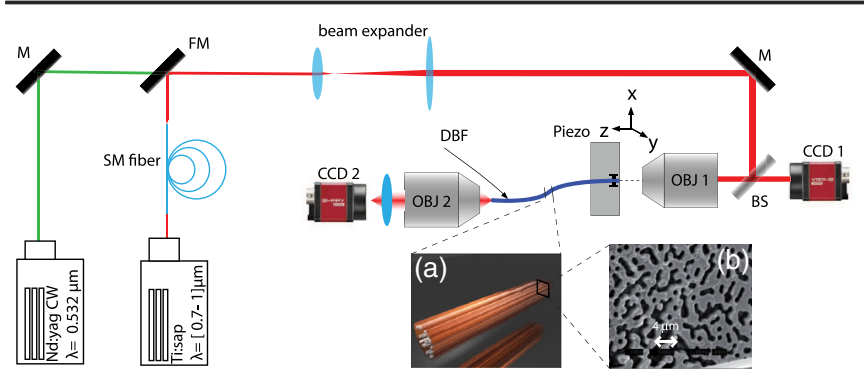

FIG. 2. Sketch of the experimental setup: The light from a ND:YAG or a Ti: sapphire laser is coupled to the fiber by using objective OBJ 1 . The backreflected light is then visualized by the camera CCD 1 through the beam splitter BS in order to perform the fine-tuning of the alignment. The piezoelectric devices control the laser injection location. The transmitted light is collected by the objective OBJ 2 and imaged on camera CCD 2 with a magnification of 50. In (a), a sketch of the fiber is reported, while in (b), a magnified image of the fiber tip surface is shown, where polymethylmethacrylate appears dark and polystyrene white.

localization length in the fiber. Thus, we estimated it by determining the square root of the spatial variance of the intensity distribution. Averaging is performed by scanning the input facet with a three-axis piezoelectric motor sustaining the fiber. The experimental setup together with a sketch of the fiber geometry is shown in Fig. 2. A more detailed description of our experiment can be found in the Supplemental Material [37].

In Fig. 1(b), we show our data for the localization length $\xi_{\infty}$ (full circles) averaged over all modes and three samples as a function of the incident-laser wavelength. It can be seen that there is no change with the wavelength in the range $0.55 \mu \mathrm{m} \leq \lambda \leq 1.05 \mu \mathrm{m}$. Our interpretation is that this discrepancy is due to the inadequacy of the potentialtype stochastic wave equation.

But why do the results of the two descriptions, which are both supposed to arise from Maxwell's equation with spatially varying permittivity, differ from each other? For deriving the wave equations in the presence of an inhomogeneous permittivity $\epsilon(\mathbf{r})$, one can either solve for the electrical field $\mathbf{E}(\mathbf{r}, t)(\mathrm{PT})$ or divide through $\epsilon(\mathbf{r})$ and then solve for the magnetic field $\mathbf{B}(\mathbf{r}, t)$ (MT):

$$
\begin{aligned}
\frac{\epsilon(\mathbf{r})}{c_{0}^{2}} \frac{\partial^{2}}{\partial t^{2}} \mathbf{E}(\mathbf{r}, t) & =-\nabla \times \nabla \times \mathbf{E}(\mathbf{r}, t) \mathrm{PT} \\
& =\nabla^{2} \mathbf{E}-\nabla(\nabla \cdot \mathbf{E}(\mathbf{r}, t)), \\
\frac{\partial^{2}}{\partial t^{2}} \mathbf{B}(\mathbf{r}, t) & =-\nabla \times \frac{c_{0}^{2}}{\epsilon(\mathbf{r})} \nabla \times \mathbf{B}(\mathbf{r}, t) \mathrm{MT} .
\end{aligned}
$$

In the absence of free charges but in the presence of a spatially fluctuating dielectric constant, we get for the divergence of the electric field

$$
\nabla \cdot \mathbf{E}=-\frac{1}{\epsilon_{0}} \nabla \cdot \mathbf{P}=-\frac{1}{\epsilon_{0}} \nabla \cdot\left[\epsilon(\mathbf{r})-\epsilon_{0}\right] \mathbf{E}
$$

from which follows [38]

$$
\nabla \cdot \mathbf{E}=-\frac{1}{\epsilon(\mathbf{r})} \mathbf{E} \cdot \nabla \epsilon(\mathbf{r}) \neq 0
$$

Obviously, in the first paper using the PT approach [12] and the following literature [13,14,18,26-28,31], the divergence of $\mathbf{P}$ (which describes the frozen-in displacement charges) had been tacitly assumed to be zero (with the exception of Refs. $[39,40])$. We believe that this is the origin of the discrepancy of the two theories.

We further check the consistency or otherwise of the two approaches by applying the theory of the nonlinear sigma model of localization to the stochastic Helmholtz equations (2). Wegner [4] realized that the nonlinear sigma model of planar ferromagnetism obeys the same scaling of the coupling constant with the length scale $L$ as the conductance $g$ of electrons in the scaling theory of localization [2], namely,

$$
\frac{d g}{d \ln L}=g \beta(g)=(d-2) g-c
$$

where $d$ is the dimensionality and $c$ is a constant of order unity. Later, a rigorous mapping of the field-theoretical representation of the configurationally averaged Green's functions to a generalized nonlinear sigma model was established [5,6,41]. This was then adapted to classical sound waves [15,21], and, using the PT approach, to electromagnetic waves [12-14]. For $d=2$, the solution of Eq. (6) is

$$
g(L)=g\left(L_{0}\right)-c \ln \left(L / L_{0}\right)
$$

where $L_{0}$ is the reference length scale; i.e., $g$ scales always towards zero. The localization length $L \equiv \xi_{\infty}$ is the length at which $g=1[6,15]$, and $g\left(L_{0}\right)=g_{0}$ is the reference conductance.

The nonlinear-sigma-model theory provides us via a saddle-point approximation with a nonperturbative way to calculate the reference conductance, which is related to the scattering mean free path $\ell_{0}(\mathrm{E})$. Within this saddle-point approximation (self-consistent Born approximation $[15,21]), g_{0}(\mathrm{E})$ and $\ell_{0}(\mathrm{E})$ are given in terms of a complex self-energy function $\Sigma(s)=\Sigma^{\prime}(\mathrm{E})+i \Sigma^{\prime \prime}(\mathrm{E})$ with complex spectral parameter $s=\mathrm{E}+i \eta, \eta \rightarrow 0$,

$$
\begin{aligned}
& \ln \xi_{\infty}(\mathrm{E}) \propto g_{0}(\mathrm{E})=\frac{\mathrm{E}+k_{0}^{2} \Sigma^{\prime}(\mathrm{E})}{k_{0}^{2} \Sigma^{\prime \prime}(\mathrm{E})} \quad \mathrm{PT}, \\
& \ln \xi_{\infty}(\mathrm{E}) \propto g_{0}(\mathrm{E})=\frac{1-\Sigma^{\prime}(\mathrm{E})}{\Sigma^{\prime \prime}(\mathrm{E})} \quad \mathrm{MT} .
\end{aligned}
$$

The function $\Sigma(s)$ obeys the self-consistent equation 


$$
\begin{aligned}
& \Sigma(s)=\gamma \frac{k_{0}^{2}}{q_{c}^{2}} \int_{0}^{q_{c}} q d q \mathrm{G}(q, s) \quad \mathrm{PT}, \\
& \Sigma(s)=\gamma \frac{1}{q_{c}^{2}} \int_{0}^{q_{c}} q d q q^{2} \mathrm{G}(q, s) \quad \mathrm{MT},
\end{aligned}
$$

with the disorder parameter $\gamma=\left\langle(\Delta \tilde{\epsilon})^{2}\right\rangle(\mathrm{PT}), \gamma=$ $\left\langle(\Delta \tilde{\epsilon})^{-2}\right\rangle(\mathrm{MT})$ and the averaged one-particle Green's functions

$$
\begin{aligned}
& \mathrm{G}(q, s)=\frac{1}{-s-k_{0}^{2} \Sigma(s)+q^{2}} \equiv \frac{1}{-k_{\Sigma}(s)^{2}-q^{2}} \quad \mathrm{PT}, \\
& \mathrm{G}(q, s)=\frac{1}{-s+q^{2}[1-\Sigma(s)]} \equiv \frac{[1-\Sigma(s)]^{-1}}{-k_{\Sigma}(s)^{2}-q^{2}} \quad \mathrm{MT},
\end{aligned}
$$

where we have introduced a complex wave number $k_{\Sigma}(s)=k_{\Sigma}^{\prime}(\mathrm{E})+i k_{\Sigma}^{\prime \prime}(\mathrm{E})$ in an obvious way. The imaginary part of this quantity is related to the scattering mean free path by $\ell_{0}(\mathrm{E})=1 / 2 k_{\Sigma}(\mathrm{E})^{\prime \prime}$, and we obtain for both descriptions (see Refs. [15,21] and the Supplemental Material [37])

$$
g_{0}(\mathrm{E})=k_{\Sigma}^{\prime}(\mathrm{E}) \ell_{0}(\mathrm{E}) .
$$

The upper $q$ integration limit is given by the correlation parameter $q_{c}=2 \pi / L_{c}[16,42]$, where $L_{c}$ is the disorder correlation length (approximate diameter of the grains with different permittivities). We determined this parameter in our samples by an image-processing correlation analysis to be $q_{c}=8 \mu \mathrm{m}^{-1}$. We use this parameter as the length scale in our field-theoretical calculations.

Again, we note that in Eqs (8)-(10), $k_{0}$ enters as an external parameter within the PT approach, whereas this is not the case in the MT approach. This results in a universal localization curve $\xi(\mathrm{E})$ for the MT theory. In contrast to this, the PT theory predicts different correlation length functions $\xi\left(k_{0}, \mathrm{E}\right)$, as demonstrated in Fig. 3, where we show the reference conductance $g_{0}(\mathrm{E})$, which is proportional to the logarithm of the localization length $\xi_{\infty}(E)$, for the two alternative theories against the spectral parameter $\mathrm{E}$.

From the function $\xi(E) \propto \exp \left\{g_{0}(\mathrm{E})\right\}$, the distribution density of localization lengths can be calculated via its inverse function $\mathrm{E}(\xi)$ as

$$
P(\xi) \propto P(\mathrm{E}(\xi))|\partial \mathrm{E} / \partial \xi| .
$$

$P(\mathrm{E})$ is the distribution of the spectral parameter, which is constant up to the maximum value $\mathrm{E}_{\max }=k_{0}^{2} \sin \left(\theta_{\max }\right)^{2}$ given by the numerical aperture. In our setup, $\theta_{\max } \approx 50^{\circ}$ corresponding to a maximum value of $\mathrm{E} / q_{c}^{2} \approx 1$. If the excited-mode range covers the whole $\xi(\mathrm{E})$ spectrum (which is the case for our experimental setup), MT theory, therefore, predicts that the distribution density $P(\mathrm{E})$, and, therefore, also the average $\langle\xi\rangle$ should not depend on the

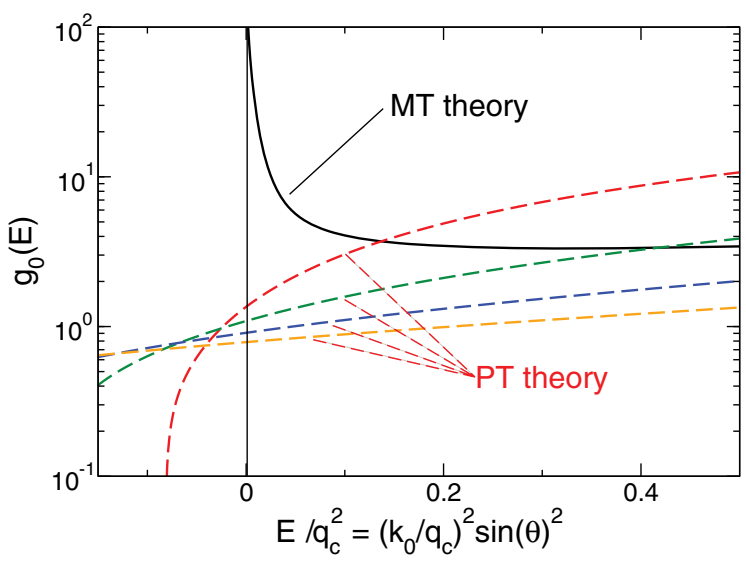

FIG. 3. Reference conductance $g_{0}(\mathrm{E}) \propto \ln \xi_{\infty}(\mathrm{E})$, where $\xi_{\infty}(E)$ is the localization length for the modulus-type description (continuous black line) and for the potential-type description (dashed lines) with four different wavelengths (from top to bottom) $\lambda=1 \mu \mathrm{m}$ (red), $\lambda=0.75 \mu \mathrm{m}$ (green), $\lambda=0.6 \mu \mathrm{m}$ (blue), $\lambda=0.5 \mu \mathrm{m}$ (orange). For both calculations, we used the disorder parameter $\gamma=0.2$. The correlation parameter $k_{c}$ has been determined from the spatial distribution of the dielectric constants (see Fig. 2) by an image-processing correlation analysis to be $k_{c}=8 \mu \mathrm{m}^{-1}$.

wavelength $\lambda=2 \pi / k_{0}$. This is in agreement with our experiment shown in Fig. 1(b). On the other hand, PT theory predicts the distribution and, hence, the average of $\xi$ to be $\lambda$ dependent in agreement with the simulations of Karbasi et al. [31] shown in Fig. 1(a) but in strong disagreement with our measurements. We emphasize again that this additional frequency dependence comes from the factor $k_{0}^{2} \propto \lambda^{-2}$ in the effective potential of the PT version of Eq. (2).

Within the modulus-type description, the reference conductance diverges as $E^{-1}$ for $E \rightarrow 0$. For the mean free path, one obtains $\ell_{0}(\mathrm{E}) \propto \mathrm{E}^{-3 / 2}$, which is equivalent to a two-dimensional Rayleigh law. As pointed out in the beginning, this absence of scattering for rays entering the sample exactly in the $z$ direction indicates that the sample is lengthwise transparent, as it should be. The Rayleigh law can also be written as $\ell_{0} \propto\left(\lambda_{\perp} / L_{c}\right)^{3}$, where $\lambda_{\perp}=\lambda / \sin \theta$ is the transverse wavelength. So, if $\lambda_{\perp}$ becomes much larger than the grain size $L_{c}$, there is no scattering and, hence, no localization.

On the contrary, within the potential-type description, the sample is predicted to be opaque in the $z$ direction, and the Rayleigh law is absent. It is even seen from Fig. 3 that the spectral scattering intensity of the potential-type model extends into the negative $E$ range, rendering the spectrum unstable. Stability, i.e., the restriction of the spectrum to positive values, is, however, required for disordered bosonic systems $[43,44]$.

Let us comment also on the three-dimensional (3D) case. Almost the entire previous literature on Anderson localization of light $[8-14,17-19]$ is based on the potential-type 
approach. So, it appears to be important to see which conclusions concerning the wavelength dependence of the localization characteristics within the MT picture will be changed going from $2 \mathrm{D}$ to $3 \mathrm{D}$.

As emphasized above, within the MT description, the only dependence of the 2D localized modes on the wavelength is via the spectral parameter $\mathrm{E}$. Therefore, the distribution of localization lengths, which is given by the function $\xi(\mathrm{E})$, does not depend on the wavelength (if it is not truncated by the numerical-aperture cutoff). In 3D (where the spectral parameter is $\omega^{2}$ ), the situation is very different: The "natural" state of a wave function in the presence of disorder is the delocalized one. One has only the chance for Anderson localization to occur near a band edge caused by the geometry of the sample-like in photonic crystals [9]. The limiting frequency (mobility edge), if it exists, is situated very near to this band edge, so the localized states will be limited to a small frequency window. The distribution of localization lengths and, hence, its average will depend on the edges of this window. Therefore, the observed localization characteristics certainly will depend on the wavelength of the light applied to probe localization.

A comparison between the localization properties of PT and MT (discretized) scalar wave equations has, in fact, already been done by Pinski et al. $[45,46]$. They find that both approaches lead to an Anderson-localization transition at the upper band edge and that, in both cases, the critical properties are the same as those of the electronic Anderson transition. However the phase diagrams (disorder strength vs spectral parameter) of the two approaches differ appreciably. In particular, in the PT case, the range of localized states within the phase diagram is somewhat larger than in the MT case.

We believe that it is worthwhile to reconsider the existing PT-based theoretical predictions of 3D Anderson localization using the MT theory in order to check which of the existing predictions remain valid and which not.

Another important difference in the results of the two approaches is as follows: In the PT approach, the influence of the disorder diminishes if the frequency $\omega=c k_{0}$ goes toward zero. This means that the permittivity $\tilde{\epsilon}=\tilde{\epsilon}(\omega=0)$ is predicted not to be affected by the disorder. On the other hand, the MT theory predicts that the permittivity is altered by the disorder, in agreement with the classical theory of Bruggeman [47] and experimental data $[48,49]$.

We see that the potential-type approach leads to general inconsistencies and, in particular, to an inconsistency with our measured localization data. This is not the case with the modulus-type description, which leads to a positive spectrum, predicts transparency in the $z$ direction, and is consistent with our measured data on transversely localized optical samples. Thus, we are convinced that we have now established a sound theoretical fundament for further work on Anderson localization of light.
M. L. acknowledges "Fondazione CON IL SUD," Grant "Brains2south," Project "Localitis."

[1] P. W. Anderson, Phys. Rev. 109, 1492 (1958).

[2] E. Abrahams, P. W. Anderson, D. C. Licciardello, and T. V. Ramakrishnan, Phys. Rev. Lett. 42, 673 (1979).

[3] P. A. Lee and T. V. Ramakrishnan, Rev. Mod. Phys. 57, 287 (1985).

[4] F. Wegner, Z. Phys. B 35, 207 (1979).

[5] L. Schäfer and F. Wegner, Z. Phys. B 38, 113 (1980).

[6] A. J. McKane and M. Stone, Ann. Phys. (N.Y.) 131, 36 (1981).

[7] F. Evers and A. D. Mirlin, Rev. Mod. Phys. 80, 1355 (2008).

[8] S. John, Phys. Today 44, 32 (1991).

[9] Photonic Crystals and Light Localization in the 21st Century, edited by C. M. Soukoulis (Springer-Science + Business Media, Dordrecht, 2001).

[10] D. S. Wiersma, Nat. Photonics 7, 188 (2013).

[11] M. Segev, Y. Silverberg, and D. N. Christodoulides, Nat. Photonics 7, 197 (2013).

[12] S. John, Phys. Rev. Lett. 53, 2169 (1984).

[13] S. John, Phys. Rev. B 31, 304 (1985).

[14] S. John, Phys. Rev. Lett. 58, 2486 (1987).

[15] S. John, H. Sompolinsky, and M. J. Stephen, Phys. Rev. B 27, 5592 (1983).

[16] S. John and M. J. Stephen, Phys. Rev. B 28, 6358 (1983).

[17] J. Kroha, C. M. Soukoulis, and P. Wölfle, Phys. Rev. B 47, 11093 (1993).

[18] P. Sheng, Introduction to Wave Scattering, Localization and Mesoscopic Phenomena (Springer, Heidelberg, 2006).

[19] P. Wölfle and D. Vollhardt, Int. J. Mod. Phys. B 24, 1526 (2010).

[20] W. Schirmacher, E. Maurer, and M. Pöhlmann, Phys. Status Solidi (c) 1, 17 (2004).

[21] W. Schirmacher, Europhys. Lett. 73, 892 (2006).

[22] W. Schirmacher, G. Ruocco, and T. Scopigno, Phys. Rev. Lett. 98, 025501 (2007).

[23] S. E. Skipetrov and J. H. Page, New J. Phys. 18, 021001 (2016).

[24] T. Sperling, L. Schertel, M. Ackermann, G. J. Aubry, C. M. Aegerter, and G. Maret, New J. Phys. 18, 013039 (2016).

[25] S. S. Abdullaev and F. K. Abdullaev, Sov. J. Radiofizika 23, 766 (1980).

[26] H. De Raedt, A. Lagendijk, and P. de Vries, Phys. Rev. Lett. 62, 47 (1989).

[27] T. Schwartz, G. Bartal, S. Fishman, and M. Segev, Nature (London) 446, 52 (2007).

[28] S. Karbasi, C. R. Mirr, P. G. Yarandi, R. J. Frazier, K. W. Koch, and A. Mafi, Opt. Lett. 37, 2304 (2012).

[29] A. Mafi, Adv. Opt. Photonics 7, 459 (2015).

[30] G. Ruocco, B. Abaie, A. Mafi, W. Schirmacher, and M. Leonetti (to be published).

[31] S. Karbasi, C. R. Mirr, R. J. Frazier, P. G. Yarandi, K. W. Koch, and A. Mafi, Opt. Express 20, 18692 (2012).

[32] C. Ganter and W. Schirmacher, Phys. Rev. B 82, 094205 (2010)

[33] S. Karbasi, C. R. Mirr, R. J. Frazier, P. G. Yarandi, K. W. Koch, and A. Mafi, Opt. Express 20, 18692 (2012). 
[34] S. Karbasi, R. J. Frazier, C. R. Mirr, K. W. Koch, and A. Mafi, J. Visualized Exp. 77, e50679 (2013).

[35] M. Leonetti, S. Karbasi, A. Mafi, and C. Conti, Phys. Rev. Lett. 112, 193902 (2014).

[36] S. Karbasi, K. W. Koch, and A. Mafi, Opt. Express 21, 305 (2013).

[37] See Supplemental Material at http://link.aps.org/ supplemental/10.1103/PhysRevLett.120.067401 for a more comprehensive description of the experimental setup and the field-theoretical calculations.

[38] B. E. A. Saleh and M. C. Teich, Fundamentals of Photonics (Wiley, New York, 1991), Chap. 5, Eq. (5.2-14).

[39] B. Abaie and A. Mafi, Phys. Rev. B 94, 064201 (2016).

[40] S. Karbasi, K. W. Koch, and A. Mafi, J. Opt. Soc. Am. B 30, 1452 (2013).

[41] A. Houghton, A. Jevicki, R. Kenway, and A. M. M. Pruisken, Phys. Rev. Lett. 45, 394 (1980).
[42] W. Schirmacher, B. Schmid, C. Tomaras, G. Viliani, G. Baldi, G. Ruocco, and T. Scopigno, Phys. Status Solidi (c) 5, 862 (2008).

[43] V. Gurarie and J. T. Chalker, Phys. Rev. B 68, 134207 (2003).

[44] T. Lück, H.-J. Sommer, and M. R. Zirnbauer, J. Math. Phys. (N.Y.) 47, 103304 (2006).

[45] S. Pinski, W. Schirmacher, and R. A. Römer, Europhys. Lett. 97, 16007 (2012).

[46] S. Pinski, W. Schirmacher, T. Whall, and R. A. Römer, J. Phys. Condens. Matter 24, 405401 (2012).

[47] D. A. G. Bruggeman, Ann. Phys. (Berlin) 416, 636 (1935).

[48] K. T. Chung, A. Sabo, and A. P. Pica, J. Appl. Phys. 53, 6867 (1982).

[49] A. V. Goncharenko, Phys. Rev. E 68, 041108 (2003). 